whether S-1 plus cisplatin, when compared with S-1 alone, prolongs overall survival in patients with advanced gastric cancer.

A total of 298 chemotherapy-naive patients (aged 20-74 years) with recurrent or unresectable carcinoma of the stomach were randomly allocated to receive either $\mathrm{S}-1$ plus cisplatin $(n=148)$ or S-1 alone $(n=150)$ at 38 centers in Japan between 26 March 2002 and 30 November 2004. During follow-up (median duration 34.7 months), 129 (87\%) of the patients in the S-1 plus cisplatin group and $139(93 \%)$ of the patients receiving S-1 alone died. The median overall survival was significantly longer in patients assigned to S-1 plus cisplatin than in those assigned to $\mathrm{S}-1$ alone (13 months vs 11 months; $P=0.04$ ). In comparison with patients receiving $\mathrm{S} 1$ alone, a greater proportion of patients receiving the combination regimen responded to treatment (31\% vs $54 \%$ ). There were more grade 3 or 4 adverse events (e.g. leucopenia, neutropenia, anemia, nausea and anorexia) in patients receiving the combination, but no treatment-related deaths were reported in either group.

The authors suggest that S-1 plus cisplatin might become a standard first-line treatment for advanced gastric cancer in Japan.

Original article Koizumi W et al. (2008) S-1 plus cisplatin versus S-1 alone for first-line treatment of advanced gastric cancer (SPIRITS trial): a phase III trial. Lancet Oncol 9: 215-221

\section{PSA velocity does not aid long-term prediction of prostate cancer incidence}

Elevated serum prostate-specific antigen (PSA) levels can indicate the presence of prostate cancer, although PSA levels are also elevated in some nonmalignant conditions, which affects the reliability of prostate cancer prediction. PSA levels rise sharply in patients with aggressive prostate cancer, and a recent study suggested that the rate of increase (PSA velocity) could predict life-threatening prostate cancer 10-15 years before diagnosis. Ulmert and colleagues evaluated data from the Malmö Preventative Medicine population-based study to compare the accuracy of a single PSA measurement versus PSA velocity in the long-term prediction of prostate cancer diagnosis.

The group analyzed archived blood samples, taken from 4,907 men (aged 33-50 years) during the period 1974-1986. Each participant supplied two samples, collected 6 years apart. In this unscreened Swedish population, the 443 prostate cancers were largely detected only when they became symptomatic. Increases in PSA levels and PSA velocity were observed in these men, up to 20 years before diagnosis. The predictive value of PSA velocity was slightly lower than that of the most recent PSA measurement (concordance 0.712 vs 0.771 ). A combined model including both PSA velocity and PSA level did not improve the accuracy of prediction.

The observed lack of additional predictive value for PSA velocity indicates that PSA levels do not increase sharply before prostate cancer diagnosis but rise gradually over many years, even in those men who present with advanced cancer.

Original article Ulmert D et al. (2008) Long-term prediction of prostate cancer: prostate-specific antigen (PSA) velocity is predictive but does not improve the predictive accuracy of a single PSA measurement 15 years or more before cancer diagnosis in a large, representative, unscreened population. J Clin Oncol 26: 835-841

\section{Immunopanel of five biomarkers provides superior definition of basal-like breast cancer}

The basal-like subtype of breast cancer is commonly identified by the triple-negative phenotype (TNP) on immunohistochemical work-up, as basal-like cancers do not express estrogen receptor, progesterone receptor, or HER2. Despite widespread use of the TNP approach for identifying basal-like breast cancers, this method has never been formally validated against the gold standard for basal-like tumor identification-gene profiling. The addition of EGFR and cytokeratin $5 / 6$ as positive markers (the 'five-marker' method) could increase the specificity of immunohistochemical panels for the basal-like subtype. Cheang et al. compared the prognostic values of these two models in 4,046 primary breast tumor samples.

Among the 3,744 samples that could be unambiguously classified, the TNP scheme defined 639 (17\%) samples as basal-like, while the five-marker scheme assigned 336 (9.0\%) samples to the core-basal subtype (i.e. negative for estrogen receptor, progesterone receptor and HER2 and positive for either EGFR or cytokeratin 5/6) and 303 (8\%) to the five-marker 